\title{
Steady-state Operation Area of VSC-HVDC Converter Station Connecting Renewable Energy Cluster by Isolated Network
}

\author{
Wenyuan Xian ${ }^{1,2}$, Ran Ding ${ }^{3}$, Ying Qiao ${ }^{2, *}$, Zongxiang $\mathrm{Lu}^{2}$, Shangqiang $\mathrm{Li}^{2}$, and Hong Lin ${ }^{1}$ \\ ${ }^{1}$ School of Electrical Engineering, Xinjiang University, Urumqi 830047, China \\ ${ }^{2}$ State Key Lab of Control and Simulation of Power Systems and Generation Equipment (Department of Electrical Engineering, Tsinghua \\ University), Haidian District, Beijing 100084, China \\ ${ }^{3}$ State Grid Jibei Electric Power Company, Xicheng District, Beijing 100053, China
}

\begin{abstract}
When the large-scale renewable power island is connected to VSC-HVDC transmission system, we should figure out the steady-state operation area of VSC-HVDC converter station. Based on the equivalent model of renewable energy island, the constraints of VSC-HVDC converter station are analyzed, and a fast method for calculating steady-state operation area of converter station is presented in this paper. The influence of equivalent line impedance of renewable energy island, transformer ratio of converter station and voltage setting value of grid-connected point on steady-state operation area of converter station is analyzed. And the three-dimensional view of the steady-state operation area of the converter station under different grid-connected voltage is depicted.
\end{abstract}

\section{Introduction}

VSC-HVDC transmission technology has obvious advantages in clean energy grid-connected, island power supply, offshore platform power supply and other technical fields([1],[2]). In recent years, more and more large-scale offshore and onshore renewable energy clusters are transmitted to load centers through VSCHVDC transmission[3]. By the analysis of existing VSCHVDC projects, it is seen that VSC-HVDC transmission technology has the ability to deliver isolated network renewable energy cluster.

The renewable energy island is decoupled from the $\mathrm{AC}$ power grid when it is delivered through the VSCHVDC transmission system. The AC side control mode of the converter station is constant voltage/frequency control, which provides voltage and frequency reference for the renewable energy AC island system. Converter station equals as a voltage source, providing reactive power and voltage support for AC islands[4]. In such a system, the steady-state operation of converter station is one of the important factors that restrict the maximum power of renewable energy island[5]. Therefore, Research on constraints and influencing factors of steady-state operation area of converter station is a great significance about the steady-state operation of the whole renewable energy island delivery system. At the same time, it can provide guidance for the optimization of reactive power and voltage in renewable energy island.

At present, some achievements have been made in the study of the steady-state operation area of VSCHVDC transmission system. The stable operation region of VSC-HVDC system connected with weak AC system is studied, and the stability constraints under various operation modes are given[6]. [7] analyzed the influence of AC network strength on the steady-state characteristics of VSC-HVDC transmission system. [8] studied the influence of short circuit ratio of AC system, shunt reactive compensation capacitor, converter transformer capacity and converter station capacity on stable operation range of converter station. But the particularity of the system is not considered. In practical engineering application, [9] proposed that the steadystate operation area of the converter station can be calculated under the maximum and minimum $\mathrm{AC}$ voltage in Zhoushan multi-terminal DC(VSC-MTDC) project, taking into account the tap position and the range of DC voltage. [10] analyzed the operation characteristics of the renewable energy isolated network connected to the VSC-HVDC power grid, considered the constraints that the steady-state operation interval should met, and describes the steady-state operation area by traversal method. And the influence of various operations on the steady-state operation area is analyzed, such as connecting transformer tap, the VSC-HVDC reference voltage, the impedance characteristics of equivalent distance of the renewable energy cluster and the VSC-HVDC converter station. However, traversal method needs to traverse all power points in unit circle of PQ power, which takes too long. And in the converter station, the voltage power operation characteristic curve changes from two-dimensional curve to threedimensional space of P-Q-V. Therefore, it is inappropriate to measure the safety margin of converter station simply by using two-dimensional voltage-power characteristic curve.

\footnotetext{
* Corresponding author: qiaoying@tsinghua.edu.cn
} 
In summary, in view of the shortcomings of existing research, this paper aims at the renewable energy island network access to VSC-HVDC transmission system. Considering the internal parameter constraints of converter stations and the voltage constraints of renewable energy cluster, a fast method for calculating the steady-state operation region of converter stations is proposed. Finally, the influence of equivalent line impedance of renewable energy island, transformer ratio of converter station and voltage setting value of gridconnected point on steady-state operation area of converter station is analyzed. And the three-dimensional voltage-power operation characteristic curve is depicted, the influence of grid-connected voltage on steady-state operation area of converter station is more intuitively depicted.

\section{System model}

Fig. 1. shows the typical topology of renewable energy cluster integrated by islanded VSC-HVDC system. The radial network is composed of $220 \mathrm{kV}$ AC. The renewable energy island accesses to the lower voltage level. There is no traditional synchronous unit in the renewable energy island. Each wind farm, photovoltaic power stations are directly assembled to the gridconnected point (PCC), and their outputs are then sent out through the converter station.

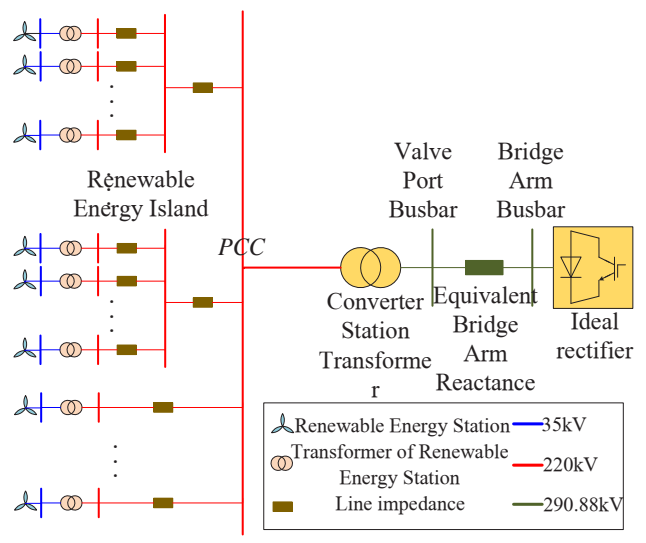

Fig. 1. Typical topology of renewable energy cluster integrated by islanded VSC-HVDC system.

Since converter station is the subject of our research, the renewable energy cluster can be static equivalent. The equivalent model of island access converter station can be obtained as shown in figure 2. In figure $2, P_{s}+j Q_{s}$ represents output of the power source and $Z_{s}$ is the equivalent impedance. The equivalent power source can be obtained from the total active and reactive power output of each renewable energy station, and the equivalent impedance can be calculated according to equation (1).

In figure $2, P_{c c}+j Q_{c c}$ is the power of grid-connected point, $P_{v}+j Q_{v}$ is the power of converter station transformer, $P_{\triangle}+j Q_{\triangle}$ is the power of converter station, $U_{s}$ is the voltage of equivalent power source, $U_{p c c}$ is the voltage of grid-connected point, $U_{v}$ is the voltage of converter station transformer, $U_{\triangle}$ is the voltage of converter station, $X_{T}$ is the impedance of transformer in converter station, $X_{L O}$ is the impedance of bridge arm in converter station, $k$ is the variable Ratio of Transformer in Converter Station.

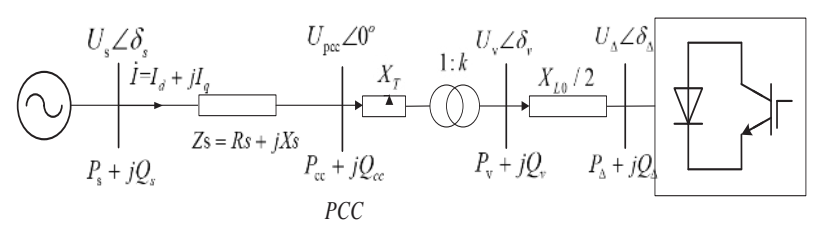

Fig. 2. Equivalent topology of renewable energy group integrated by islanded VSC-HVDC system.

$$
Z_{s}=\left(P_{l o s s}+j Q_{l o s s}\right)\left(\frac{\dot{U_{p c c}}}{P_{c c}+j Q_{c c}}\right)^{*}\left(\frac{\dot{U_{p c c}}}{P_{c c}+j Q_{c c}}\right)=\left(P_{l o s s}+j Q_{l o s s}\right) \frac{U_{p c c}^{2}}{P_{c c}^{2}+Q_{c c}^{2}}
$$

Where $(\cdot)^{*}$ denotes the conjugation. Under different output conditions of renewable energy cluster, the system losses are different, and so the equivalent impedance is also different.

\section{Characterization method of steady- state operating area of converter sta- tion}

\subsection{Constraints of steady-state operating area of converter station}

The steady-state operation area of converter station is a set of system operation points that satisfy the constraints on capacity of converter station. When the operation point of converter station is in the steady-state operation area, the system security can be guaranteed. The steadystate operation area of converter station is generally depicted in two-dimensional plane with active and reactive power in abscissa and longitudinal coordinates. Assume that the AC bus voltage $U_{p c c}$ of a VSC-HVDC converter station is known at a certain time and the power flowing into the converter station is $P_{c c}+j Q_{c c}$, the operating range of the renewable energy VSC-HVDC system should satisfy the following constraints: tion

a. Capacity constraint of VSC-HVDC converter sta-

$$
\sqrt{P_{c c}^{2}+Q_{c c}^{2}} \leq S_{c c N}
$$

where $S_{c c N}$ is the rated capacity of converter station.

b. Capacity constraints of transformers in VSCHVDC converter station

$$
\sqrt{P_{v}^{2}+Q_{v}^{2}} \leq S_{v N}
$$

where $S_{v N}$ is the rated capacity of transformer in converter station.

c. AC current constraint of VSC-HVDC converter station 


$$
\frac{\sqrt{P_{c c}^{2}+Q_{c c}^{2}}}{k U_{p c c}} \leq I_{c c N}
$$

where $I_{c c N}$ is the rated current of converter station.

d. DC current constraint of VSC-HVDC converter station

$$
-I_{d c \max } \leq \frac{P_{c c}}{U_{d c N}} \leq I_{d c \max }
$$

where $I_{d c \max }$ is the maximum DC current of converter station, $U_{d c N}$ is the DC side rated voltage.

e. Modulation ratio constraints of VSC-HVDC converters

$$
m_{\min } \leq m=\frac{2 \sqrt{2} U_{\Delta}}{\mu U_{\mathrm{dc}}} \leq m_{\max }
$$

where $m$ is the modulation ratio of VSC-HVDC Converter, $m_{\max }$ is the maximum modulation ratio, $m_{\max }$ is the minimum modulation ratio, $m_{\max }$ equals $1.15 . \mu$ is the utilization rate of DC voltage, which equals 0.866 . $U_{d c}$ is the DC bus voltage.

f. Transformer ratio constraint in converter station

$$
k_{\min } \leq k \leq k_{\max }
$$

where $k$ is the transformer ratio of converter station. $k_{\max }$ equals $1.075, k_{\min }$ equals 0.925 .

g. Voltage constraints of systems

$$
U_{\min } \leq U_{s}, U_{p c c}, U_{v} \leq U_{\max }
$$

where $U_{\min }$ is the minimum voltage constraints of systems, which equals 0.97 . $U_{\max }$ is the maximum voltage constraints of systems, which equals 1.07.

h. Constraints on operation range of renewable energy stations

$$
\left\{\begin{array}{l}
Q_{s \min } \leq Q_{s} \leq Q_{s \max } \\
P_{s \min } \leq P_{s} \leq P_{s \max }
\end{array}\right.
$$

where $Q_{s}, Q_{s \min }$ and $Q_{s \max }$ are respectively the total reactive power output, the minimum total reactive power output and the maximum total reactive power output of the renewable energy station. $P_{s}, P_{s \min }$ and $P_{s \max }$ are respectively the minimum and maximum total effective output of renewable energy station.

i. Constraints of system power flow equation

$$
\left\{\begin{array}{l}
P_{i}=U_{i} \sum_{j=1}^{N} U_{j}\left(G_{i j} \cos \theta_{i j}+B_{i j} \sin \theta_{i j}\right) \\
Q_{i}=U_{i} \sum_{j=1}^{N} U_{j}\left(G_{i j} \sin \theta_{i j}-B_{i j} \cos \theta_{i j}\right)
\end{array}\right.
$$

where $P_{i}$ and $Q_{i}$ are the injection active power and reactive power of node $i, U_{i}$ is the voltage value of node $i$, $G_{i j}$ and $B_{i j}$ are the branch conductance and branch admittance between node $i$ and node $j$, and indicate the phase difference between node $i$ and node $j$.

\subsection{Description method of steady-state opera- ting area of converter station}

Obtaining the set of power points $\left(P_{c c}, Q_{c c}\right)$ satisfying the constraint of formula $(2) \sim(10)$ is the base for analysis on steady-state operating area. We can consider the constraint of formula (2) as a unit power circle and the steady-state operation area of the converter station must be within this unit circle. Therefore, we can find the power point satisfying the constraints from the boundary of the unit circle inward. The specific steps are as follows:

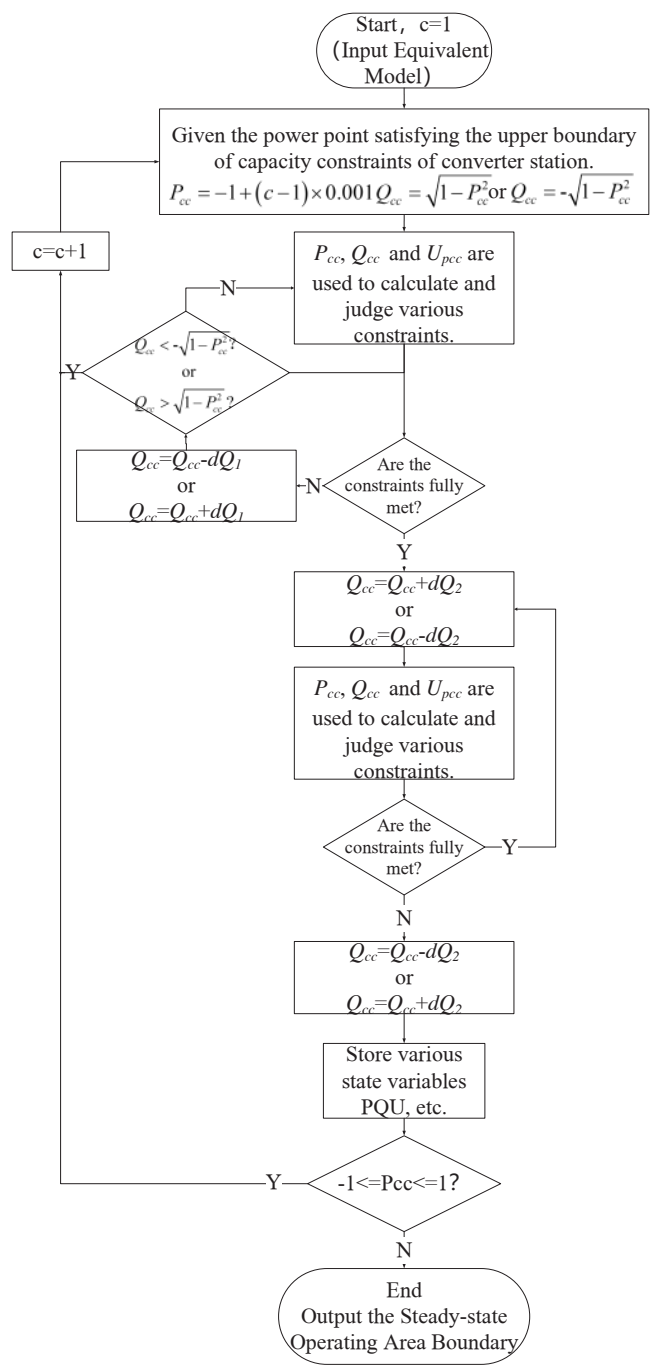

Fig. 3. Flow charts for describing the steady-state operation area of converter station.

Step 1. Input the equivalent model of the system.

Step 2. The power point satisfying the capacity constraint boundary of converter station is given.

Step 3. The electrical quantities in the system can be calculated by equation (10). Then each electrical quantity is substituted into equation (3) $\sim(9)$ to determine whether all constraints are satisfied at the same time. If not, $Q_{c c}$ subtracts step $d Q_{l}$ downward $\left(Q_{c c}\right.$ adds step $d Q_{1}$ up) (The length of $d Q_{1}$ should not be too large, otherwise it is possible to cross the steady-state operation area.), and judges whether the value of $Q_{c c}$ exceeds the lower boundary of the unit circle (If it exceeds, go back to step 2, if it does not, go back to step 
3.). If satisfied, it proves that the current power point has been in the steady-state operation area of the converter station, and the next step is taken.

Step 4. Because $d Q_{I}$ is set relatively large, the power point at this time may cross the boundary of safe and stable operation area, so $Q_{c c}$ needs to increase (reduce) $d Q_{2} \quad\left(d Q_{1}\right.$ is greater than $d Q_{2}$.) upward (down). The electrical quantities in the system can be calculated by equation (10). Then each electrical quantity is substituted into equation (3) $\sim(9)$ to determine whether all constraints are satisfied at the same time.

Step 5. If the constraints are met, it means that the power point is still in the steady-state operation area, and return to step 4. If not, then $Q_{c c}$ subtracts (adds) $d Q_{2}$, and stores various state variables corresponding to the current power point.

Step 6. Judge whether the value of $P_{c c}$ is in the unit circle, if it is, return to step 2. If it is not, end the cycle, and describe the upper boundary of safe and stable operation area of converter station with all stored power points.

The steady-state operation area of the converter station can be obtained by the steps displayed in Fig. 3 . It should be noted that in order to improve the calculation speed, we do not use ergodic method, and we rather set the corresponding step size $d Q_{1}$ and $d Q_{2}$. The set of power points satisfying constraints can be found more quickly by changing step size.

\section{Case study}

The shape, size and location of the steady-state operation area of the converter station are affected by the constraints shown in formula (2). For a certain renewable energy island VSC-HVDC delivery system, most of its system parameters are fixed, such as the total power output limit and the modulation ratio of converter station, and so forth. However, the equivalent line impedance of renewable energy islands, the voltage setting value of converter station and the converter conversion ratio may vary during the operation of the system, thus changing the size of other electrical quantities of the system. Therefore, the sensitivity analysis method is used to explore the influence of these three factors on the shape, size and relative position of the steady-state operation area of the system. The unit circle is the capacity constraint of the converter station.

\subsection{Equivalent line impedance of renewable energy island}

According to equation (1), the equivalent line impedance of renewable energy island is related to the total power output of renewable energy station. Fig. 4. shows the steady-state operation area diagram of the grid-connected point under different equivalent impedance. It can be found that with the increase of equivalent impedance, the steady-state operation area decreases gradually. This shows that the magnitude of equivalent impedance has a great influence on the steady-state operation area.
In fact, for a certain renewable energy island system (just like the system mentioned in this paper), the equivalent line impedance will not change much with the power output of the station. As shown in Fig. 5. When the output power of all renewable energy station changes from $0 \%$ to $100 \%$, the maximum change of equivalent impedance resistance is not more than 0.0001 , and the maximum change of reactance is not more than 0.003 .

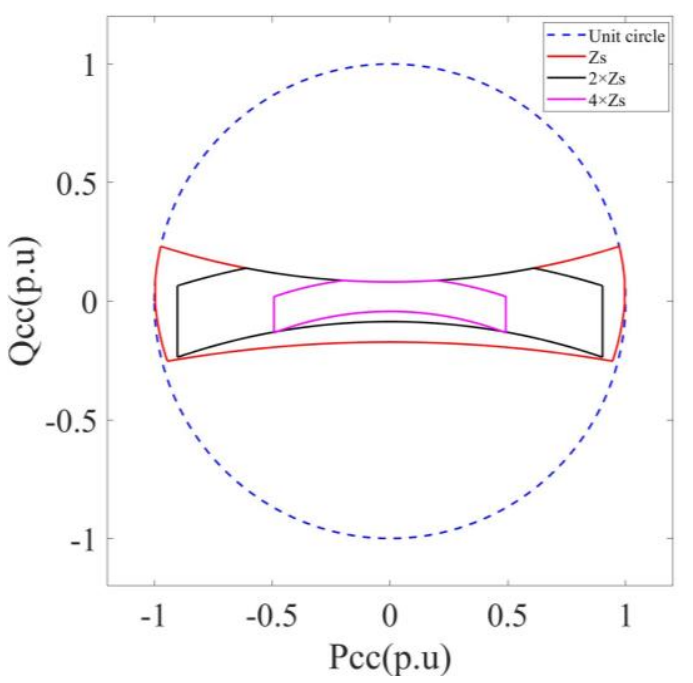

Fig. 4. Steady-state operation area of converter station under different equivalent impedances.

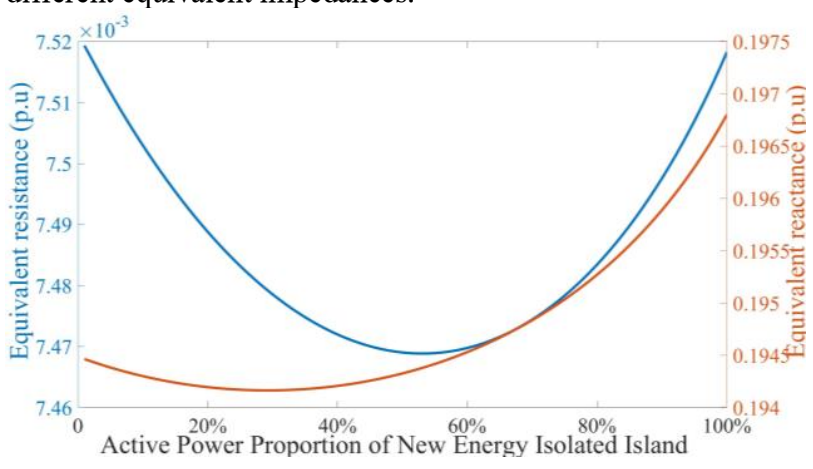

Fig. 5. Renewable energy island equivalent impedance value with different active power output ratio.

\subsection{Transformer ratio of converter station}

Under fixed islanding equivalent impedance and gridconnected voltage setting, changing transformer ratio of converter station, the steady-state operation area of converter station under different situations can be obtained, as shown in Fig. 6.

With the change of transformer ratio in converter station, its steady-state operation area will change for both shape and size. The reason is that when the transformer ratio of converter station changes, the reactive power distribution of the system will change, so the position of steady-state operation area will shift up and down. It can be seen that the setting of transformer ratio in converter station is also very important for the steady-state operation of converter station. 


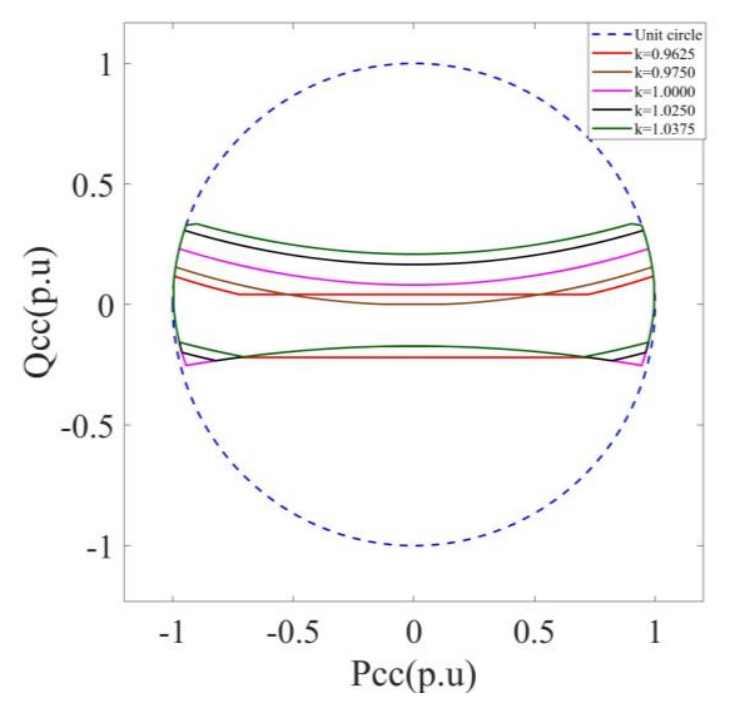

Fig. 6. Steady-state operation area of converter station under different transformer ratio of converter station.

\subsection{Voltage setting value of grid-connected po- int}

Under fixed islanding equivalent impedance and transformer ratio of converter station, we changed gridconnected voltage setting, and the steady-state operation area of converter station under different situations can be obtained, as shown in Fig. 7.

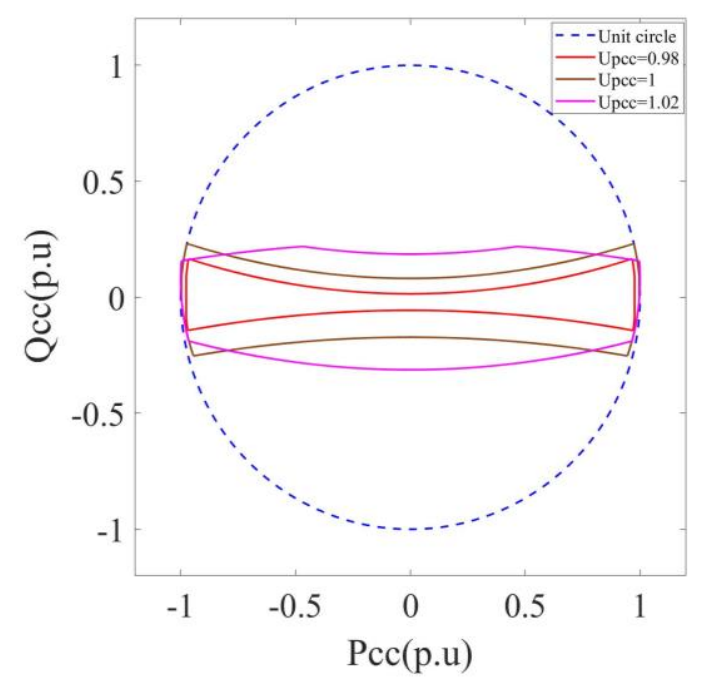

Fig. 7. Steady-state operation area of converter station under different grid-connected voltage settings.

With the increase of voltage setting value, the shape of steady-state region changes obviously. Fig. 8 shows a three-dimensional view of the steady-state operation area of the converter station under different grid-connected voltages. The influence of grid-connected voltage on steady-state operation area can be seen more intuitively from the figure. When the voltage is at a certain value, the maximum steady-state operation area can be obtained. With this voltage as the starting point, increasing or decreasing the voltage will reduce the steady-state operation area.

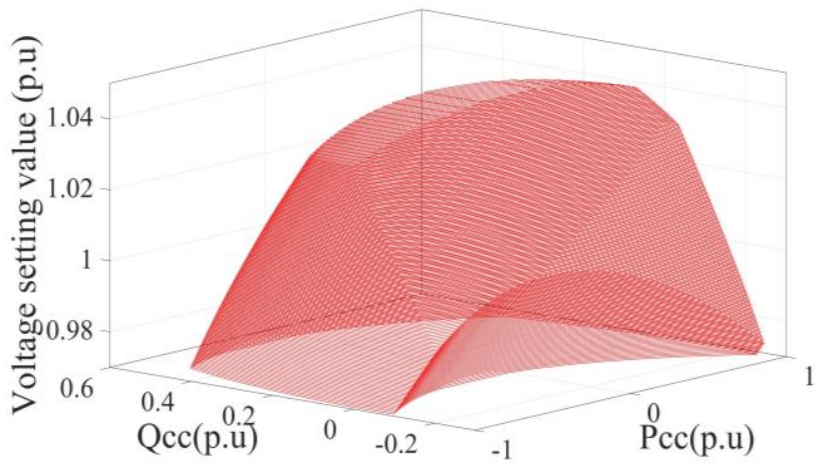

Fig. 8. Three-dimensional view of the steady-state operation area of the converter station under different grid-connected voltage.

\section{Discussion and conclusion}

In this paper, we study on steady-state operation area of VSC-HVDC converter station connecting renewable energy cluster by isolated network. The conclusions are as follows:

Based on the analysis on the constraints for the steady-state operation area of the converter station, a method is proposed to characterize the steady-state operation area of the converter station. This method can depict the steady-state operation area of converter station more quickly.

Although the magnitude of equivalent impedance has an influence on the steady-state operation area, for a certain renewable energy island system, the equivalent line impedance will not change much with the power output of the station. Therefore, for a certain renewable energy island system, only the transformer ratio of converter station and voltage setting value of gridconnected point have great influence on the steady-state operation area of converter station.

From the three-dimensional diagram, we can see the influence of grid connected voltage on the steady-state operation area more intuitively.

This work was supported by State Grid Corporation Science (SGJB0000TKJS1801210).

\section{References}

1. Kang, H. S. , and A. Yazdani . [IEEE 2016 North American Power Symposium (NAPS) - Denver, CO, USA (2016.9.18-2016.9.20)] 2016 North American Power Symposium (NAPS) - Enhancement of power system stability with VSC-HVDC transmission. North American Power Symposium IEEE, 2016:1-5.

2. Guangfu Tang, Zhiyuan He, Hui Pang. Research, Application and Development of VSC-HVDC Engineering Technology. Automation of Electric Power Systems, 2013, 37(15): 3-14.

3. Korompili, Asimenia, Q. Wu, and H. Zhao. Review of VSC HVDC connection for offshore wind power 
integration. Renewable and Sustainable Energy Reviews, 59(2016):1405-1414.

4. Yongning Chi, Zhankui Zhang, Yan Li, et al. Development of Large-scale Wind Power Grid Integration and Technical Standard. North China Electric Power, 2017, (3):1-7.

5. Linlin $\mathrm{Wu}, \mathrm{Man} \mathrm{Xu}$, Hui Liu, et al. Optimal Voltage Control Strategy for Islanded Aggregated New Energy System Connecting by VSC-HVDC. Journal of Global Energy Interconnection, 2018,1(02):112119.

6. Sheng Liu, Zheng XU. Study on Stable Operating Region of VSC-HVDC Connected to Weak AC Systems. Proceedings of the CSEE, 2016,36(01):133-144.
7. Zhou J Z, Gole A M. VSC transmission limitations imposed by ac system strength and ac impedance characteristics. In: 10th IET Int. Conf. on AC-DC Power Transmission. 2012: 1-6.

8. Jing Zhang, et al. Steady-State Operating Range of MMC-HVDC. Electric Power Construction 36.3(2015):1-6.

9. Yanan Li, Weiyong Jiang, Shifeng Yu, et al. System Design of Zhoushan Multi-terminal VSC-HVDC Transmission Project. High Voltage Eng ineering, 2014, 40( 8):2490-2496.

10. Ying Liu, Linlin $\mathrm{Wu}$, Man $\mathrm{Xu}$, et al. Study on Steady-state Operation Region of VSC-HVDC Converter Station Connecting New Energy Cluster by Isolated Network. North China Electric Power, 2017(06):8-13 